\title{
Characteristics and Management of Candidaemia Episodes in an Established Candida auris Outbreak
}

\author{
Juan V. Mulet Bayona ${ }^{1, *, \dagger}$, Nuria Tormo Palop ${ }^{1,+}$, Carme Salvador García ${ }^{1,+}$, \\ Paz Herrero Rodríguez ${ }^{2}$, Vicente Abril López de Medrano ${ }^{2}$, Carolina Ferrer Gómez ${ }^{3}$ \\ and Concepción Gimeno Cardona ${ }^{1,4}$ \\ 1 Microbiology Department, Consorcio Hospital General Universitario de Valencia, 46014 Valencia, Spain; \\ tormo_nur@gva.es (N.T.P.); salvador_cargar@gva.es (C.S.G.); concepcion.gimeno@uv.es (C.G.C.) \\ 2 Infectious Diseases Department, Consorcio Hospital General Universitario de Valencia, 46014 Valencia, \\ Spain; paz.herrero@goumh.umh.es (P.H.R.); abril_viclop@gva.es (V.A.L.d.M.) \\ 3 Anestesiology and Reanimation Department, Consorcio Hospital General Universitario de Valencia, \\ 46014 Valencia Spain; ferrer_cargoma@gva.es \\ 4 Microbiology Department, University of Valencia, 46100 Burjassot, Spain \\ * Correspondence: mulet_jua@gva.es \\ + These authors contributed equally to this work.
}

Received: 29 July 2020; Accepted: 28 August 2020; Published: 30 August 2020

\begin{abstract}
The multi-resistant yeast Candida auris has become a global public health threat because of its ease to persist and spread in clinical environments, especially in intensive care units. One of the most severe manifestations of invasive candidiasis is candidaemia, whose epidemiology has evolved to more resistant non-albicans Candida species, such as C. auris. It is crucial to establish infection control policies in order to control an outbreak due to nosocomial pathogens, including the implementation of screening colonisation studies. We describe here our experience in managing a C. auris outbreak lasting more than two and a half years which, despite our efforts in establishing control measures and surveillance, is still ongoing. A total of 287 colonised patients and 47 blood stream infections (candidaemia) have been detected to date. The epidemiology of those patients with candidaemia and the susceptibility of $C$. auris isolates are also reported. Thirty-five patients with candidaemia $(74.5 \%)$ were also previously colonised. Forty-three patients $(91.5 \%)$ were hospitalised $(61.7 \%)$ or had been hospitalised $(29.8 \%)$ in the ICU before developing candidaemia. Antifungal therapy for candidaemia consisted of echinocandins in monotherapy or in combination with amphotericin B or isavuconazole. The most common underlying disease was abdominal surgery $(29.8 \%)$. The thirty-day mortality rate was $23.4 \%$ and two cases of endophtalmitis due to C. auris were found. All isolates were resistant to fluconazole and susceptible to echinocandins and amphotericin B. One isolate became resistant to echinocandins two months after the first isolate. Although there are no established clinical breakpoints, minimum inhibitory concentrations for isavuconazole were low $(\leq 1 \mu \mathrm{g} / \mathrm{mL})$.
\end{abstract}

Keywords: Candida auris; candidaemia; fungi; yeast; outbreak; multidrug-resistant; colonisation; surveillance

\section{Introduction}

An increase in the prevalence of Candida bloodstream infections (candidaemia) and a shift in the epidemiology have been observed in recent years, especially since the emergence of the multidrug-resistant yeast Candida auris [1]. C. auris was first identified in 2009, from the auditory canal of a Japanese patient [2] and, since then, it has been reported in a large variety of body parts and in the six continents of the world [3]. This yeast is considered a growing menace to global health for several reasons, which include its resistance to multiple commonly used antifungals, its problematic 
identification in the laboratory and its facility to spread among patients, causing nosocomial outbreaks, especially in intensive care units (ICU) [4]. Different organisations, such as Centers for Disease Control and Prevention (CDC) or the European Centre for Disease Prevention and Control (ECDC), claim that there is an emergency in using reliable methods to identify Candida spp. isolates to the species level and encourage hospitals to do screening colonisation studies in order to control the outbreaks caused by C. auris [5,6]. In our setting, there is an ongoing C. auris outbreak since October 2017 [7], and, despite the implementation of different control measures (e.g., periodic screening for C. auris surveillance), we still report cases, showing the difficulty in eradicating this nosocomial pathogen.

The aim of this study is, first, to describe the C. auris outbreak that is currently ongoing in our setting and the measures established in order to control it. Secondly, we analyse the evolution in the Candida species distribution causing candidaemia in our setting since 2011 and the clinical and epidemiological characteristics of all patients diagnosed with C. auris candidaemia, as well as the antifungal susceptibility of the isolates.

\section{Results}

\subsection{Description of the Outbreak}

In September 2017, C. auris was detected for the first time in our hospital in the urine culture of a patient. One month later, in October 2017, a second case was detected in a blood culture of another patient, being therefore the first case of candidaemia. Since then, screening for $C$. auris colonisation is part of the infection control practices in the ICU, where the outbreak is located. Screening is performed before a patient is admitted in the ICU and once a week until hospital discharge. When a colonised or infected patient is discharged to a general ward, surveillance is also performed, and when a case is detected outside the ICU, surveillance cultures are performed to contacts in the ward. Together with the screening of patients, other measures were implemented, such as the isolation of cases, patient cohorting, the reduction of health workers in contact with colonised patients, the decolonisation of patients with clorhexidine solutions and environmental cleaning with hydrogen peroxide solutions (Oxivir ${ }^{\circledR}$ ). Environmental surveillance is also performed in the ICU once a year or when an increase in the number of cases is produced, and C. auris has been isolated from mattresses. tables, computers and an emergency button. The entire ICU was also cleaned with a more concentrated hydrogen peroxide solution following the environmental cultures.

A total of 287 colonised patients and 47 candidaemia episodes (35 of them were also previously colonised) have been detected since the beginning of the outbreak (Figure 1). Our ICU includes 31 beds and it is divided in two sections: a general ICU and a cardiac ICU. Due to the large amount of cases and the long evolution of the outbreak, the C. auris outbreak already affects the entire ICU. In July 2018, despite the implementation of infection control practices and surveillance cultures, an increase was observed with four new patients with candidaemia (Figure 1). After that, new episodes of candidaemia continued to be detected but at a lower frequency. However, in April 2020, the incidence of colonisation and candidaemia increased again, coinciding with the novel coronavirus SARS-CoV-2 pandemic in Spain (Figure 1). Recently, a PCR assay has been introduced in our laboratory for the detection of C. auris in surveillance samples, which is expected to reduce the response time from $24-48 \mathrm{~h}$ to $1 \mathrm{~h}$, compared to conventional culture (unpublished data). 


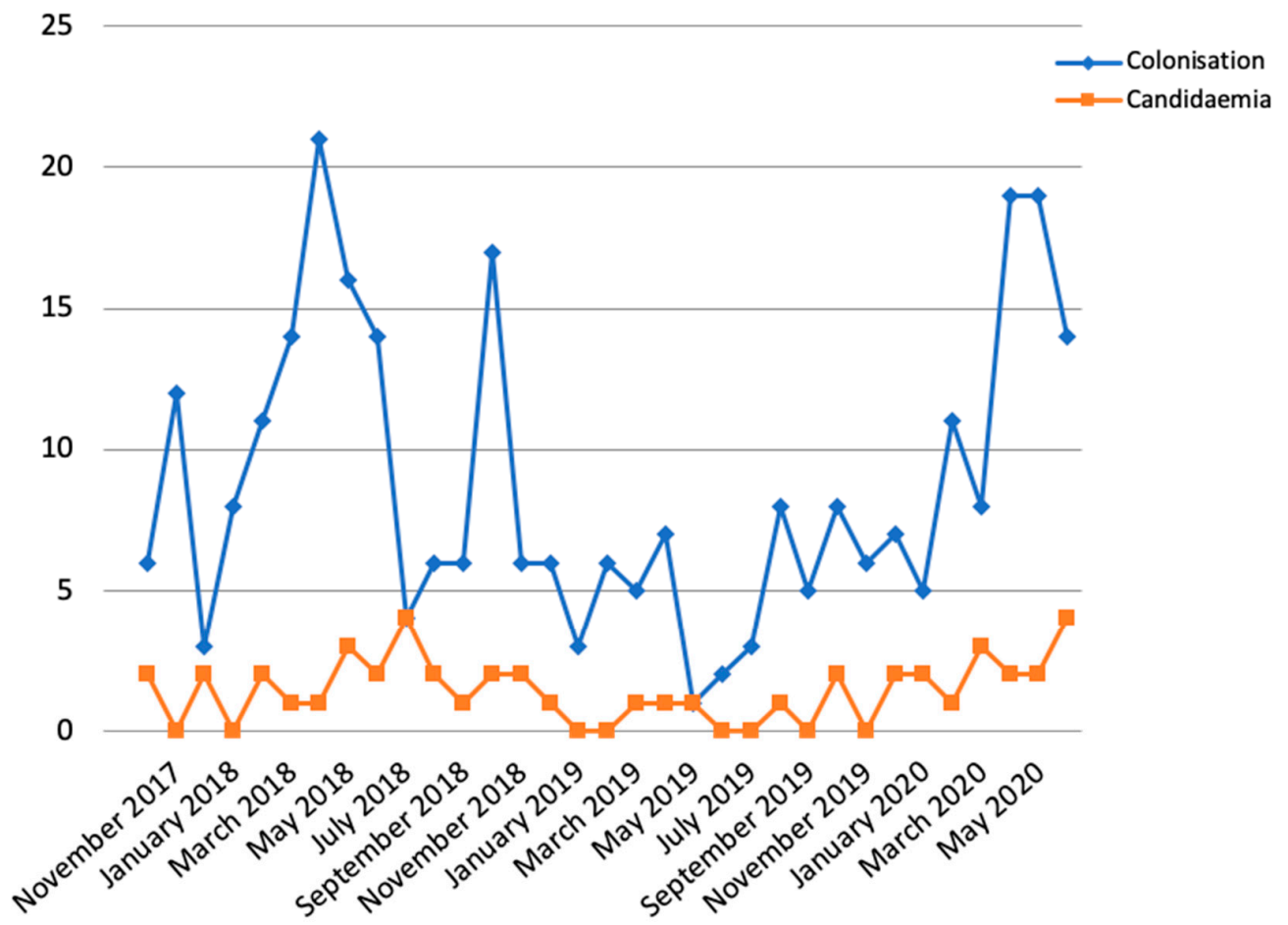

Figure 1. New colonised patients and candidaemia episodes from October 2017 to June 2020.

\subsection{Characteristics of C. auris Candidaemias}

The evolution of species distribution causing candidaemia since 2011 in our setting is shown in Figure 2. Although C. albicans has always been the most isolated species, a high proportion of more resistant Candida species (e.g., C. glabrata, C. parapsilosis) are also usually isolated in all studied years. In 2017, when C. auris emerged, this pathogen rapidly spread and caused several episodes of candidaemia. Especially remarkable is the large increase in candidaemia in 2018, the first year since the outbreak was established, becoming the year with most candidaemia episodes reported. In the first half of 2020, a large number of candidaemia episodes have also been produced, especially due to C. auris.

\section{Candaemia episodes by year and species}

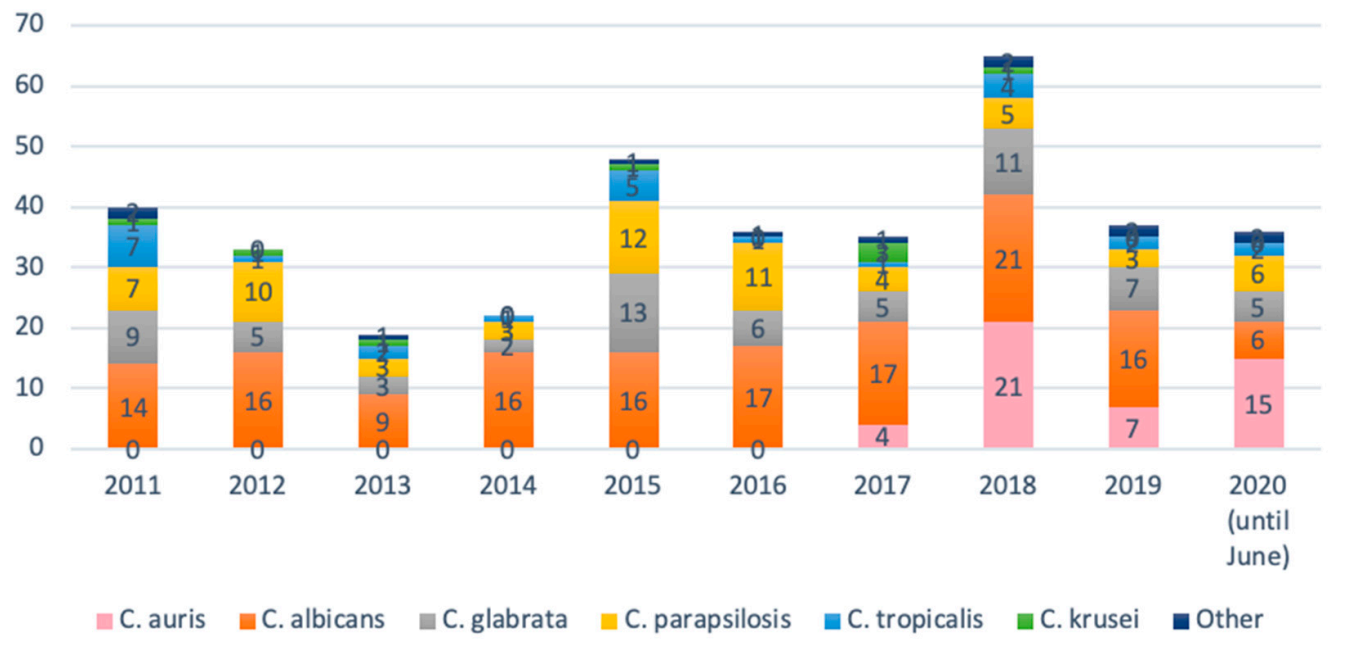

Figure 2. Species distribution in candidaemias produced in our setting since 2011 to June 2020. 
The median length of hospital stay before the candidaemia episode was 29 days (IQR 19.5-39.5). The median total length of hospital stay was 70 days (IQR 39.5-85). A total of 91.5\% of patients (43/47) were hospitalised or had been hospitalised in the ICU before developing candidaemia. In 29 patients $(61.7 \%)$, candidaemia was detected when the patient was in the ICU, while in 14 patients $(29.8 \%)$, candidaemia was detected once the patient was discharged from the ICU, to a general ward. Four patients admitted to a general ward (8.5\%) were never hospitalised in the ICU but developed C. auris candidaemia. However, those cases could not be related to other cases produced in a general ward. The median length of ICU stay before the candidaemia episode was 28 days (IQR: 19.5-40.5) and the median total length of the ICU stay was 29 days (IQR: 16-49.5). Only one patient developed candidaemia in the first week of stay in the ICU, five patients in the second week and nine patients in the third week. The remaining 28 patients took four or more weeks since admission to the ICU until the candidaemia episode. Epidemiological and clinical characteristics, antifungal treatment and outcome of 47 patients with $C$. auris candidaemia are summarised in Table 1 . In seven patients, C. auris was isolated along with another Candida species: three were mixed with Candida albicans, two with Candida tropicalis, one with Candida krusei, and one with C. albicans and Candida parapsilosis. Antifungal treatment was administered until two weeks after a negative blood culture, and it consisted of echinocandins in monotherapy $(46.8 \%)$ or in combination with amphotericin B (25.5\%) or isavuconazole (21.3\%), when this most recent azole was available in our hospital. The median duration of the antifungal treatment was 21 days (IQR: 15-25).

Table 1. Epidemiological and clinical characteristics, antifungal treatment and outcome of C. auris candidaemia.

\begin{tabular}{|c|c|}
\hline Characteristics & $\mathrm{N}=47$ \\
\hline Age, mean (SD) & $61(16.3)$ \\
\hline Male & $35(74.5 \%)$ \\
\hline APACHE II, mean (SD) & $15.1(7.0)$ \\
\hline Candida score, mean (SD) & $1.48(1.18)$ \\
\hline No risk & $9(19.2 \%)$ \\
\hline Low risk & $23(48.9 \%)$ \\
\hline High risk & $15(31.9 \%)$ \\
\hline Charlson comorbidity index, mean (SD) & $3.0(2.1)$ \\
\hline \multicolumn{2}{|l|}{ Underlying disease } \\
\hline Abdominal surgery & $14(29.8 \%)$ \\
\hline Digestive disease, not surgical & $9(19.1 \%)$ \\
\hline Cardiovascular disease & $7(14.9 \%)$ \\
\hline Neurovascular disease & $5(10.6 \%)$ \\
\hline COVID-19 & $5(10.6 \%)$ \\
\hline Respiratory disease, not COVID-19 & $3(6.4 \%)$ \\
\hline Otorhinolaringologic disease & $2(4.3 \%)$ \\
\hline Politrauma & $2(4.3 \%)$ \\
\hline \multicolumn{2}{|l|}{ Risk factors } \\
\hline Diabetes & $13(27.7 \%)$ \\
\hline Malignancy & $12(25.5 \%)$ \\
\hline Immunosuppression & $2(4.3 \%)$ \\
\hline Renal replacement therapy & $8(17.0 \%)$ \\
\hline Total parenteral nutrition & $7(14.9 \%)$ \\
\hline Sepsis & $10(21.3 \%)$ \\
\hline Surgery ( $<30$ days before candidaemia) & $24(51.1 \%)$ \\
\hline Mechanical ventilation & $29(61.7 \%)$ \\
\hline Central venous catheter & $39(83.0 \%)$ \\
\hline Urinary catheter & $38(80.9 \%)$ \\
\hline
\end{tabular}


Table 1. Cont.

\begin{tabular}{|c|c|}
\hline Characteristics & $\mathrm{N}=47$ \\
\hline ICU stay more than two weeks & $33(70.2 \%)$ \\
\hline Previous antibiotic treatment & $43(91.5 \%)$ \\
\hline \multicolumn{2}{|l|}{ Days administered: } \\
\hline$<7$ days & $3(6.4 \%)$ \\
\hline 7 to 14 days & $13(27.7 \%)$ \\
\hline$>15$ days & $27(57.4 \%)$ \\
\hline Carbapenem use & $25(53.2 \%)$ \\
\hline Previous antifungal treatment (administered for more than 5 days) & $14(29.8 \%)$ \\
\hline Fluconazole & $2(4.3 \%)$ \\
\hline Voriconazole & $1(2.1 \%)$ \\
\hline Echinocandin & $11(23.4 \%)$ \\
\hline \multicolumn{2}{|l|}{ Therapeutic measures } \\
\hline Antifungal treatment & $44(93.6 \%)$ \\
\hline Echinocandin in monotherapy & $22(46.8 \%)$ \\
\hline Echinocandin plus Amphotericin B & $12(25.6 \%)$ \\
\hline Echinocandin plus Isavuconazole & $10(21.3 \%)$ \\
\hline \multicolumn{2}{|l|}{ Other measures } \\
\hline Central venous catheter removal & $33(70.2 \%)$ \\
\hline Urinary catheter replacement or removal & $3(6.4 \%)$ \\
\hline \multicolumn{2}{|l|}{ Outcome } \\
\hline 30-day mortality & $11(23.4 \%)$ \\
\hline \multicolumn{2}{|l|}{ Complications } \\
\hline Endophtalmitis & $2(4.3 \%)$ \\
\hline Recurrence of candidaemia & $7(14.9 \%)$ \\
\hline Persistence of candidaemia & $6(12.8 \%)$ \\
\hline
\end{tabular}

Surveillance cultures were conducted in all patients except four: the first case and three patients because they were not hospitalised in the ICU. From the performed cultures, 35 were positive $(81.4 \%)$, 9 for both axillary-rectal and pharyngeal samples (25.7\%), 24 only for the axillary-rectal sample $(68.6 \%)$ and two only for the pharyngeal sample (5.7\%). The median time since admittance to the ICU to a positive colonisation sample was 14 days (IQR 9.5-19.5). Six patients had a positive colonisation sample in the first week since they were admitted to the ICU (17.1\%), one of which was already hospitalised in a general ward. Thirteen patients were positive to colonisation in the second week after they were admitted to the ICU (37.1\%), two of which were already hospitalised in a general ward. Eight patients were positive to colonisation in the third week $(22.9 \%)$ and the remaining eight patients were positive in the fourth or more weeks after their admittance to the ICU $(22.9 \%)$, most of which were already hospitalised in a general ward. One patient was positive at the admission screening in the ICU, but this patient had a prior stay in the ICU registered from a month before. The median time since a positive colonisation sample to the candidaemia episode was 16 days (IQR 8-25).

Antifungal susceptibility of $C$. auris isolates is reported in Table 2. Only the first isolate of every patient was included in the table, as susceptibility did not differ greatly. However, it is noteworthy that an isolate that was resistant to echinocandins was isolated from a patient from whom a susceptible strain was isolated two months earlier. This patient presented a first episode of candidaemia associated with a permanent central venous catheter and was treated with anidulafungin for 28 days and antifungal lock of the catheter with anidulafungin until the catheter was replaced, but a second episode of candidaemia with the resistant strain was produced. 
Table 2. Antifungal susceptibility of $C$. auris isolates from blood cultures.

\begin{tabular}{ccccccccccc}
\hline Antifungal & FLZ & AFG & MCF & CFG & AMB & PSC & VRC & ITC & 5FC & ISA \\
\hline $\begin{array}{c}\text { Isolates } \\
\text { tested }\end{array}$ & 47 & 47 & 47 & 47 & 47 & 30 & 36 & 28 & 19 & 15 \\
Range & 256 & $0.06-0.25$ & $0.03-0.5$ & $0.03-0.25$ & $0.125-1$ & $0.015-0.5$ & $0.5-8$ & $0.06-0.5$ & $0.06-0.25$ & $0.03-0.5$ \\
MIC $_{50}$ & 256 & 0.125 & 0.06 & 0.125 & 0.5 & 0.125 & 4 & 0.25 & 0.125 & 0.25 \\
MIC $_{\mathbf{9 0}}$ & 256 & 0.25 & 0.125 & 0.25 & 1 & 0.25 & 8 & 0.5 & 0.25 & 0.5 \\
GM & 256 & 0.144 & 0.068 & 0.102 & 0.606 & 0.123 & 2.939 & 0.269 & 0.110 & 0.170 \\
\hline
\end{tabular}

5FC, flucytosine; AFG, anidulafungin; $\mathrm{AMB}$, amphotericin B; CFG, caspofungin; FLZ, fluconazole; GM, geometric mean MIC; ISA, isavuconazole; ITC, itraconazole; MFG, micafungin; MIC50 and MIC90, minimum inhibitory concentration required to inhibit the growth of $50 \%$ and $90 \%$ of the isolates, respectively; PSC, posaconazole; VRC, voriconazole.

\section{Discussion}

C. auris is a global public health threat because of its ease to persist and spread in a clinical environment, among other reasons. During an outbreak, patients are colonised with C. auris at several body sites, including axilla, groin, nostrils, ears and rectum, and it also has been detected in beds, tables, floors, walls, equipment and monitors [8-12]. Several measures were established in order to control the outbreak, following the recommendations of the ECDC [6], the CDC [5] and the previous experience of other established outbreaks, as the first European outbreak described in a London cardio-thoracic centre [13] or the first Spanish outbreak described in [11]. These infection control practices include the strict isolation/cohorting of cases, decolonisation with chlorhexidine, regular environmental cleaning and the implementation of screening colonisation studies in the high-risk hospital environments such as the ICU. Axilla and groin swabs are usually recommended for screening, although other body parts can be sampled if it is clinically relevant [6]. In our setting, axillary-rectal and pharyngeal swabs were selected for the screening and showed good performances, especially axillary-rectal swab $(76.7 \%$ of tested patients with candidaemia were previously positive for this sample). All these measures allowed a reduction in the frequency of the isolation of $C$. auris in both clinical samples and colonisation samples. However, two years after the outbreak started, and despite our efforts at establishing control measures and epidemiological surveillance, $C$. auris is still being isolated from colonisation samples and, what is most worrying, from blood cultures, showing that this nosocomial pathogen is very difficult to eradicate and can cause important infections.

Two peaks are observed in the evolution of the outbreak. The first one was in 2018, corresponding to the first months of the outbreak, and it could be in part because the control measures were not strictly followed by all health workers, especially in the holiday period, with some replacement staff. The second peak was in April and May 2020, coinciding with the peak of the SARS-CoV-2 pandemic in Spain. Hospitalisation rooms had to be reorganised in order to isolate patients affected by COVID-19 and that forced ignoring other nosocomial pathogens. Relaxing the control measures for $C$. auris could explain the increase in the cases (both colonisation and candidaemia) in those two periods, reinforcing the importance of infection control practices. It is important to note that the higher peak in candidaemia (July 2018) overlaps the lower peak in colonisations. This is because the patients are first colonised and approximately two weeks after develop candidaemia (the median time since ICU admittance to colonisation is 14 days and since admittance to the candidaemia episode is 28 days); therefore, a peak in colonisations is expected to produce an increase in candidaemia later.

Candidaemia is a life-threating condition in critically ill patients, which makes it crucial to understand local epidemiologic trends and the antifungal susceptibility of etiological agents. Multiple studies have shown an increase in the incidence of candidaemia and a shift to uncommon Candida species in recent years [1,14-16], including multidrug-resistant species such as C. auris, which has further complicated their management. In our setting, C. albicans is usually the predominant species, causing between a third and a half of candidaemia, followed by C. glabrata and C. parapsilosis alternating in second place until 2017, similar to other data reported in Spanish hospitals [17]. Although a clear tendency in species distribution over the years cannot be observed, what is clear is that candidaemia 
increased greatly in the first year of the outbreak, and C. auris has displaced C. glabrata and C. parapsilosis in our hospital, becoming the most isolated species in 2018, equal to C. albicans, and even exceeding C. albicans in the first half of 2020. It is noteworthy that in seven patients, C. auris was isolated along with another Candida species, which is a higher proportion than mixed non-C. auris candidaemia in the same period, which totalled five cases. Most mixed candidaemia was produced in 2018, when the larger increase in C. auris candidaemia was produced.

Several known risk factors for developing candidaemia $[18,19]$ were present in a high number of our patients affected with C. auris candidaemia, like prior antibiotic exposure $(91.5 \%)$, central venous catheter $(83.0 \%)$, mechanical ventilation $(61.7 \%)$, previous surgery $(51.1 \%)$, previous colonisation with C. auris (74.5\%) and ICU stay more than two weeks (70.2\%). Gastrointestinal disease, both surgical and non-surgical, was the most common underlying disease (48.9\%), which is also common for other Candida species candidaemia. A total of 14 patients with prophylactic antifungal treatment, 11 of them with echinocandins, developed candidaemia. Previous exposure to fluconazole or echinocandins has been associated with a higher incidence in $C$. auris candidaemia, which could be due to a selective pressure for C. auris [20]. Interestingly, age, APACHE II and Charlson comorbidity index were relatively low in the patients affected by $C$. auris candidaemia, which is also reported in a study from India comparing C. auris with non-auris Candida candidaemia [20]. This could be attributed to the profiles of patients that are usually admitted in the ICU, where the outbreak is established. As a complication of candidaemia, and despite the antifungal treatment, we detected two cases of endophtalmitis, which is less common than for other Candida species [21].

The crude mortality rate of $C$. auris candidaemia at 30 days in our series was $23.4 \%$, similar to that reported by other recent studies [22-24]. However, this is lower than the mortality rates reported in initial studies of C. auris candidaemia [25-27], ranging 30-60\% approximately, although C. auris-attributable mortality cannot be calculated from those series. The attributable mortality rate for $C$. auris candidaemia is difficult to evaluate because it affects critically ill patients with multiple comorbidities, though it should be analysed in futures studies.

C. auris is a multidrug-resistant yeast, and levels of resistance can vary between isolates, so antifungal susceptibility testing must be performed. There are no established susceptibility breakpoints, although CDC reported tentative breakpoints [28]. According to those breakpoints, all isolates were resistant to fluconazole and susceptible to echinocandins and amphotericin B. However, an isolate that was resistant to echinocandins was detected in one patient two months after the initial isolation of a sensitive strain. This might be due to a prolonged treatment with anidulafungin in the treatment of the first candidaemia episode, which exerted antimicrobial pressure. Resistance to echinocandins, amphotericin B and even pan-resistant isolates have been reported, in some cases after a prolonged treatment with these antifungals $[29,30]$. This demonstrates the need for continued surveillance, even in serial isolates from the same patient, and encourages being cautious when prescribing antifungal drugs. However, in our series, despite the intensive use of echinocandins, either in monotherapy or in combination, and also as an empirical treatment, no other strain developed resistance. MICs for posaconazole and voriconazole were variable, although the high resistance to fluconazole and to other azoles reported in the literature makes them not recommended to treat $C$. auris infections [31]. All tested isolates showed low MICs for isavuconazole $(\leq 1 \mu \mathrm{g} / \mathrm{mL})$, although there is limited evidence of the effectiveness.

The reported outbreak in our setting is one of the most important globally, with prolonged transmission over two and a half years. Although genotypic typing of the strains is still ongoing, epidemiological tracing is highly consistent with the existence of an outbreak (same hospital ward). Preliminary results with some strains suggest they belong to the South African clade, the same as other Spanish isolates [11]. 


\section{Materials and Methods}

Consorcio Hospital General Universitario de Valencia (CHGUV) is a 503-bed tertiary hospital which provides medical assistance to a population of around 400,000 people in Valencia, Spain. The evolution of the C. auris outbreak which started in October 2017 in our setting is described, as well as the infection control practices established. A screening colonisation study is performed in patients admitted to the ICU and periodically once a week. For this purpose, a pharyngeal and an axillary-rectal sample are collected and cultured in CHROMagar ${ }^{\mathrm{TM}}$ Candida (Becton, Dickinson and Company, Franklin Lakes, NJ, USA). Plates are incubated at $37^{\circ} \mathrm{C}$ for $48 \mathrm{~h}$, and lectures are done at 24 and $48 \mathrm{~h}$.

All patients with positive blood cultures for C. auris since October 2017, when the first episode was detected, to June 2020 were included. Blood samples were processed according to our routine laboratory procedure. Briefly, blood samples are incubated in BD Bactec ${ }^{\mathrm{TM}} \mathrm{FX}$ (Becton, Dickinson and Company, Franklin Lakes, NJ, USA) for 5 days or 14 days if fungi blood infection was suspected. When the sample flags positive, and the Gram stain reveals the presence of yeasts, subculture is performed in Sabouraud-Chloramphenicol and CHROMagar ${ }^{\mathrm{TM}}$ Candida. In this medium, C. auris grows in a non-specific colour of white, beige or pink, and these colonies are further identified.

Candida isolates from both blood samples and surveillance samples are further identified by matrix-assisted laser desorption/ionisation (MALDI-TOF; Bruker, Billerica, MA, USA). Susceptibility testing is carried out for candidaemia isolates by broth microdilution through Sensititre ${ }^{\mathrm{TM}}$ YestOne YO10 (Thermo Fisher Scientic, Waltham, MA, USA) for antifungals micafungin, caspofungin, anidulafungin, flucytosine, voriconazole, itraconazole, fluconazole and amphotericin B. Lastly, 15 isolates were tested through the new version of Sensititre ${ }^{\mathrm{TM}}$ (Sensititre ${ }^{\mathrm{TM}}$ YeastOne ITAMYUCC), which replaces flucytosine with isavuconazole. Tentative breakpoints from CDC were used to describe the susceptibility of the isolates to fluconazole $(\geq 32 \mu \mathrm{g} / \mathrm{mL})$, anidulafungin $(\geq 4 \mu \mathrm{g} / \mathrm{mL})$, caspofungin $(\geq 2 \mu \mathrm{g} / \mathrm{mL})$, micafungin $(\geq 4 \mu \mathrm{g} / \mathrm{mL})$ and amphotericin B ( $\geq 2 \mu \mathrm{g} / \mathrm{mL})$ [28]. Only anidulafungin, micafungin, caspofungin and amphotericin $\mathrm{B}$ were reported in all isolates because the other antifungals were not routinely used in the treatment of $C$. auris infections. Isavuconazole was tested since it was available for use in the hospital. Fluconazole is also tested as a complementary method for identification since all isolates are resistant to it. Only the first isolate from every patient is included in the analysis.

Demographic and relevant clinical data were retrospectively collected via chart review, including comorbidities, underlying diseases, risk factors, duration of hospitalisation, duration of stay in the ICU before the candidaemia episode, previous antibiotic treatment, previous antifungal treatment, therapeutic measures and clinical outcome. Recurrence of candidaemia was defined as the case when a positive blood culture was obtained after a negative one. Persistence of candidaemia was defined as the case when a positive culture was obtained after 7 days of adequate antifungal treatment.

\section{Conclusions}

The reported outbreak in our setting is one of the most important globally, with prolonged transmission over two and a half years. Our experience, as well as the experience of other hospitals reported in the literature, show that $C$. auris can persist and efficiently spread in hospital environments, being difficult to eradicate. Instauration of infection control policies and periodic screening for colonisation are essential to control an outbreak caused by C. auris. Candidaemia is one of the most worrisome conditions caused by this multidrug-resistant yeast because it usually affects critical patients. Its multidrug resistance makes it even more difficult to manage these infections, especially since C. auris has potential to become more resistant in the course of a treatment with antifungal drugs. Therefore, patients with antifungal treatment for $C$. auris should be monitored closely and susceptibility testing should always be performed.

Author Contributions: Conceptualisation, J.V.M.B., N.T.P. and C.S.G.; data curation, J.V.M.B., P.H.R., V.A.L.d.M. and C.F.G.; formal analysis, J.V.M.B., N.T.P. and C.S.G.; methodology, J.V.M.B., N.T.P. and C.S.G.; supervision, N.T.P., C.S.G. and C.G.C.; writing-original draft, J.V.M.B.; writing-review and editing, N.T.P., C.S.G. and C.G.C. All authors have read and agreed to the published version of the manuscript. 
Funding: The APC was funded by Fundación de Investigación del Hospital General Universitario de Valencia, Spain.

Conflicts of Interest: The authors declare no conflict of interest.

\section{References}

1. Van Schalkwyk, E.; Mpembe, R.S.; Thomas, J.; Shuping, L.; Ismail, H.; Lowman, W.; Karstaedt, A.S.; Chibabhai, V.; Wadula, J.; Avenant, T.; et al. Epidemiologic Shift in Candidemia Driven by Candida auris, South Africa, 2016-2017. Emerg. Infect. Dis. 2019, 25, 1698-1707. [CrossRef]

2. Satoh, K.; Makimura, K.; Hasumi, Y.; Nishiyama, Y.; Uchida, K.; Yamaguchi, H. Candida auris sp. nov., a novel ascomycetous yeast isolated from the external ear canal of an inpatient in a Japanese hospital. Microbiol. Immunol. 2009, 53, 41-44. [CrossRef] [PubMed]

3. CDC. Tracking Candida auris. Available online: https://www.cdc.gov/fungal/candida-auris/tracking-c-auris. html (accessed on 27 June 2020).

4. Forsberg, K.; Woodworth, K.; Walters, M.; Berkow, E.L.; Jackson, B.; Chiller, T.; Vallabhaneni, S. Candida auris: The recent emergence of a multidrug-resistant fungal pathogen. Med. Mycol. 2018, 57, 1-12. [CrossRef] [PubMed]

5. CDC. Candida auris. Information for Laboratorians and Health Professionals. Available online: https: //www.cdc.gov/fungal/candida-auris/health-professionals.html (accessed on 28 June 2020).

6. European Centre for Disease Prevention and Control. Candida auris in Healthcare Settings—Europe; First update, 23 April 2018; ECDC: Stockholm, Sweden, 2018.

7. Salvador García, C.; Tormo Palop, N.; Mulet Bayona, J.V.; Melero García, M.; Navalpotro Rodríguez, D.; Belda Álvarez, M.; del Remedio Guna Serrano, M.; Gimeno, C. Candida auris: Descripción de un brote. Enferm. Infecc. Microbiol. Clin. 2020, 38 (Suppl. 1), 39-44.

8. Meis, J.F.; Chowdhary, A. Candida auris: A global fungal public health threat. Lancet Infect. Dis. 2018, 18, 1298-1299. [CrossRef]

9. Kumar, J.; Eilertson, B.; Cadnum, J.L.; Whitlow, C.S.; Jencson, A.L.; Safdar, N.; Krein, S.L.; Tanner, W.D.; Mayer, J.M.; Samore, M.H.; et al. Environmental Contamination with Candida Species in Multiple Hospitals Including a Tertiary Care Hospital with a Candida auris Outbreak. Pathog. Immun. 2019, 4, 260-270. [CrossRef] [PubMed]

10. Piedrahita, C.T.; Cadnum, J.L.; Jencson, A.L.; Shaikh, A.A.; Ghannoum, M.A.; Donskey, C.J. Environmental Surfaces in Healthcare Facilities are a Potential Source for Transmission of Candida auris and Other Candida Species. Infect. Control Hosp. Epidemiol. 2017, 38, 1107-1109. [CrossRef]

11. Ruiz-Gaitán, A.; Moret, A.M.; Tasias-Pitarch, M.; Aleixandre-López, A.I.; Martínez-Morel, H.; Calabuig, E.; Salavert-Lletí, M.; Ramírez, P.; López-Hontangas, J.L.; Hagen, F.; et al. An outbreak due to Candida auris with prolonged colonisation and candidaemia in a tertiary care European hospital. Mycoses 2018, 61, 498-505. [CrossRef]

12. Jeffery-Smith, A.; Taori, S.K.; Schelenz, S.; Jeffery, K.; Johnson, E.M.; Borman, A.; Candida auris Incident Management Team; Manuel, R.; Brown, C.S. Candida auris: A review of the literature. Clin. Microbiol. Rev. 2018, 31, 1-18.

13. Schelenz, S.; Hagen, F.; Rhodes, J.L.; Abdolrasouli, A.; Chowdhary, A.; Hall, A.; Ryan, L.; Shackleton, J.; Trimlett, R.; Meis, J.F.; et al. First hospital outbreak of the globally emerging Candida auris in a European hospital. Antimicrob. Resist. Infect. Control 2016, 5, 35. [CrossRef]

14. Castanheira, M. Fungemia Surveillance in Denmark Demonstrates Emergence of Non-albicans Candida Species and Higher Antifungal Usage and Resistance Rates than in Other Nations. J. Clin. Microbiol. 2018, 56, e01907-17. [CrossRef] [PubMed]

15. Horn, D.L.; Neofytos, D.; Anaissie, E.J.; Fishman, J.A.; Steinbach, W.J.; Olyaei, A.J.; Marr, K.A.; Pfaller, M.; Chang, C.-H.; Webster, K.M. Epidemiology and Outcomes of Candidemia in 2019 Patients: Data from the Prospective Antifungal Therapy Alliance Registry. Clin. Infect. Dis. 2009, 48, 1695-1703. [CrossRef] [PubMed]

16. Lamoth, F.; Lockhart, S.R.; Berkow, E.L.; Calandra, T. Changes in the epidemiological landscape of invasive candidiasis. J. Antimicrob. Chemother. 2018, 73 (suppl. 1), i4-i13. [CrossRef] 
17. Puig-Asensio, M.; Padilla, B.; Garnacho-Montero, J.; Zaragoza, O.; Aguado, J.M.; Zaragoza, R.; Montejo, M.; Muñoz, P.; Ruiz-Camps, I.; Cuenca-Estrella, M.; et al. Epidemiology and predictive factors for early and late mortality in Candida bloodstream infections: A population-based surveillance in Spain. Clin. Microbiol. Infect. 2014, 20, O245-O254. [CrossRef] [PubMed]

18. Yapar, N. Epidemiology and risk factors for invasive candidiasis. Ther. Clin. Risk Manag. 2014, 10, 95-105. [CrossRef] [PubMed]

19. Lausch, K.R.; Søgaard, M.; Rosenvinge, F.S.; Johansen, H.K.; Boysen, T.; Røder, B.; Mortensen, K.L.; Nielsen, L.; Lemming, L.; Olesen, B.; et al. High incidence of candidaemia in a nationwide cohort: Underlying diseases, risk factors and mortality. Int. J. Infect. Dis. 2018, 76, 58-63. [CrossRef] [PubMed]

20. Rudramurthy, S.M.; Chakrabarti, A.; Paul, R.A.; Sood, P.; Kaur, H.; Capoor, M.R.; Kindo, A.J.; Marak, R.S.K.; Arora, A.; Sardana, R.; et al. Candida auris candidaemia in Indian ICUs: Analysis of risk factors. J. Antimicrob. Chemother. 2017, 72, 1794-1801. [CrossRef] [PubMed]

21. Breazzano, M.P.; Tooley, A.A.; Godfrey, K.J.; Iacob, C.E.; Yannuzzi, N.A.; Flynn, H.W. Candida auris and endogenous panophthalmitis: Clinical and histopathological features. Am. J. Ophthalmol. Case Rep. 2020, 19, 100738. [CrossRef]

22. Taori, S.K.; Khonyongwa, K.; Hayden, I.; Athukorala, G.D.A.; Letters, A.; Fife, A.; Desai, N.; Borman, A.M. Candida auris outbreak: Mortality, interventions and cost of sustaining control. J. Infect. 2019, 79, 601-611. [CrossRef]

23. Sayeed, M.A.; Farooqi, J.; Jabeen, K.; Awan, S.; Mahmood, S.F. Clinical spectrum and factors impacting outcome of Candida auris: A single center study from Pakistan. BMC Infect. Dis. 2019, 19, 384. [CrossRef]

24. Calvo, B.; Melo, A.S.A.; Perozo-Mena, A.; Hernandez, M.; Francisco, E.C.; Hagen, F.; Meis, J.F.; Colombo, A.L. First report of Candida auris in America: Clinical and microbiological aspects of 18 episodes of candidemia. J. Infect. 2016, 73, 369-374. [CrossRef] [PubMed]

25. Sarma, D.S.; Kumar, N.; Sharma, S.; Govil, D.; Ali, T.; Mehta, Y.; Rattan, A. Candidemia caused by amphotericin B and Fluconazole resistant Candida auris. Indian J. Med. Microbiol. 2013, 31, 90-91. [CrossRef] [PubMed]

26. Lee, W.G.; Shin, J.H.; Uh, Y.; Kang, M.G.; Kim, S.H.; Park, K.H.; Jang, H.-C. First three reported cases of nosocomial fungemia caused by Candida auris. J. Clin. Microbiol. 2011, 49, 3139-3142. [CrossRef]

27. Vallabhaneni, S.; Kallen, A.T.S.; Chow, N.; Welsh, R. Investigation of the First Seven Reported Cases of Candida auris, a Globally Emerging Invasive, Multidrug-Resistant Fungus-United States, May 2013-August 2016. MMWR Morb. Mortal. Wkly. Rep. 2016, 65, 1234-1237. [CrossRef]

28. CDC. Antifungal Susceptibility Testing and Interpretation. Available online: https://www.cdc.gov/fungal/ candida-auris/c-auris-antifungal.html (accessed on 6 July 2020).

29. Ostrowsky, B.; Greenko, J.; Adams, E.; Quinn, M.; O’Brien, B.; Chaturvedi, V.; Berkow, E.; Vallabhaneni, S.; Forsberg, K.; Chaturvedi, S.; et al. Candida auris Isolates Resistant to Three Classes of Antifungal Medications-New York, 2019. MMWR Morb. Mortal. Wkly. Rep. 2020, 69, 6-9. [CrossRef] [PubMed]

30. Ademe, M.; Girma, F. Candida auris: From multidrug resistance to pan-resistant strains. Infect. Drug Resist. 2020, 13, 1287-1294.

31. Sekyere, J.O. Candida auris: A systematic review and meta-analysis of current updates on an emerging multidrug-resistant pathogen. Microbiologyopen 2018, 7, e00578. [CrossRef] [PubMed]

(C) 2020 by the authors. Licensee MDPI, Basel, Switzerland. This article is an open access article distributed under the terms and conditions of the Creative Commons Attribution (CC BY) license (http://creativecommons.org/licenses/by/4.0/). 